\title{
MOTIVIC ZETA FUNCTIONS FOR PREHOMOGENEOUS VECTOR SPACES AND CASTLING TRANSFORMATIONS
}

\author{
FRANÇOIS LOESER
}

\begin{abstract}
We study the behaviour of motivic zeta functions of prehomogeneous vector spaces under castling transformations. In particular we deduce how the motivic Milnor fibre and the Hodge spectrum at the origin behave under such transformations.
\end{abstract}

\section{$\S 1$. Introduction}

1.1. Let us recall the classification of irreducible regular prehomogeneous vector spaces due to Sato and Kimura [18]. A prehomogeneous vector space over a field $k$ [18] consists of the datum $(G, X)$ of a connected linear $k$-algebraic group $G$ together with a linear representation $\rho$ of $G$ on a finite dimensional affine space $X=\mathbf{A}_{k}^{m}$ over $k$ having a Zariski dense $G$-orbit. The complement of the Zariski dense $G$-orbit is called the singular locus of the prehomogeneous vector space. When $X$ is an irreducible $G$-module, one says the prehomogeneous vector space $(G, X)$ is irreducible.

One may find a general definition of regular prehomogeneous vector spaces in [18], which, when the group $G$ is reductive, is equivalent to the condition that the singular locus is an hypersurface. From now on, except for Section 7, we shall always assume the group $G$ to be reductive. When $(G, X)$ is an irreducible regular prehomogeneous vector space, the singular locus is an irreducible hypersurface $f=0$ in $X$.

In the theory of Sato and Kimura, a fundamental role is played by castling transforms, which are defined as follows.

Let $G$ be a connected reductive $k$-algebraic group and $\rho$ be a linear representation of $G$ on $\mathbf{A}_{k}^{m}$. Write $m=r_{1}+r_{2}$. Then set $X_{1}=M_{m, r_{1}}$ and let $G \times \mathrm{SL}_{r_{1}}$ act on $X_{1}$ by

$$
\rho_{1}\left(g, g_{1}\right) x_{1}=\rho(g) x_{1}^{t} g_{1} .
$$

Received November 27, 2001.

2000 Mathematics Subject Classification: 11S90, 14L30, 32S55; 14L24. 
Similarly set $X_{2}=M_{m, r_{2}}$ and let $G \times \mathrm{SL}_{r_{2}}$ act on $X_{2}$ by

$$
\rho_{2}\left(g, g_{2}\right) x_{2}={ }^{t} \rho(g)^{-1} x_{2}{ }^{t} g_{2} \text {. }
$$

Assume $G_{j}=\operatorname{Im} \rho_{j}$ is an irreducible regular prehomogeneous vector space for $j=1,2$ with singular locus the irreducible hypersurface $f_{j}=0$. In this case one says that the prehomogeneous vector spaces $\left(G_{1}, X_{1}\right)$ and $\left(G_{2}, X_{2}\right)$ are related by a castling transformation. Furthermore, cf. 4.1, $f_{1}$ and $f_{2}$ are homogeneous polynomials whose degrees satisfy $\operatorname{deg} f_{j}=r_{j} d$, for some integer $d$. We shall say that two irreducible regular prehomogeneous vector spaces over $k$ are equivalent if, up to isomorphism, they may be included in a finite chain of irreducible regular prehomogeneous vector spaces whose consecutive terms are related by a castling transform. In each equivalence class there is a unique prehomogeneous vector space with $\operatorname{dim} X$ minimal. Such an object is called reduced in the terminology of Sato and Kimura. When $k$ is algebraically closed of characteristic zero, the set of all reduced irreducible regular prehomogeneous vector spaces has been divided into 29 types by Sato and Kimura [17]. If $k$ is of characteristic zero and one restricts to $k$-split groups, then the absolute classification restricts to a relative one.

1.2. Thus, if one wants to compute the value of some invariant for irreducible regular prehomogeneous vector spaces, it is enough: a) to understand the behaviour of the invariant under castling transformations; b) to compute the invariant explicitly for reduced prehomogeneous vector spaces. For instance, when $k=\mathbf{C}$, if one denotes by $b_{j}$ the $b$-function of $f_{j}$, we have the following relation due to Shintani (cf. [11]):

$$
b_{2}(s) \prod_{1 \leq i \leq r_{1}} \prod_{0 \leq j<d}\left(s+\frac{(i+j)}{d}\right)=b_{1}(s) \prod_{1 \leq i \leq r_{2}} \prod_{0 \leq j<d}\left(s+\frac{(i+j)}{d}\right),
$$

and the $b$-functions of all 29 types, except for one, have been tabulated by Kimura in [11] and the remaining case has been settled by Ozeki and Yano [23]. When $k$ is a $p$-adic field and $Z_{j}$ denotes Igusa's local zeta function attached to $f_{j}$, then Igusa proved in [8] the relation

$$
Z_{2}(s) \prod_{1 \leq j \leq r_{1}}\left(\frac{1-q^{-j}}{1-q^{-(d s+j)}}\right)=Z_{1}(s) \prod_{1 \leq j \leq r_{2}}\left(\frac{1-q^{-j}}{1-q^{-(d s+j)}}\right),
$$

where $q$ denotes the cardinality of the residue field and the coefficients of each polynomial $f_{j}$ are assumed to belong to the valuation ring but not all to the valuation ideal; furthermore, Igusa was able to compute $Z(s)$ for 24 out of the 29 types, see [9], [10]. 
1.3. Another important invariant of singularities of functions is the Hodge spectrum [20], [21], [22] and a natural question is to understand its behaviour under castling transformations. It is not clear to us how to answer this question using classical techniques, and the aim of the present paper is to explain how this can be achieved using motivic integration. In fact, replacing $p$-adic integration with integration on arc spaces, Denef and Loeser defined in [1], [3], [2] a motivic zeta function $Z_{f}$ for a function $f$ which is a geometric analogue of Igusa's local zeta function. The motivic zeta function $Z_{f}$ contains a great amount of information on $f$. In particular one can read off the whole Hodge spectrum of $f$ from $Z_{f}$. The main result of the present paper is Theorem 4.2 where a relation completely analogous to (1.2.2) is proved for motivic zeta functions. We then deduce the behaviour of the Hodge spectrum under castling transformations in Corollary 6.8. To prove Theorem 4.2, it would be natural to imitate Igusa's proof in the $p$-adic case as much as possible. In fact, we were not able to follow Igusa's proof to closely, since in that proof crucial use is made of properties of the Haar measure on a locally compact $p$-adic group, especially of its uniqueness, for which no complete analogue is available yet on the motivic integration side, though highly desirable ${ }^{1}$. Hence we are led to follow a more down to earth approach, relying on some direct computations in spaces of matrices, as in Lemma 5.4.

Finally, in Section 7 we give some generalizations of our results to prehomogeneous vector spaces which are no longer assumed to be irreducible and regular.

I would like to thank Jan Denef and Akihiko Gyoja for conversations that raised my interest on the questions considered in the present paper and also Akihiko Gyoja for useful remarks on the first draft of the paper.

\section{§2. Notations and conventions}

\subsection{Grothendieck groups of varieties}

We fix a field $k$ of characteristic zero. By a variety over $k$ we shall mean a separated and reduced scheme of finite type over $k$. Let $S$ be an algebraic variety over $k$. By an $S$-variety we mean a variety $X$ together with a morphism $X \rightarrow S$. The $S$-varieties form a category denoted by $\operatorname{Var}_{S}$, the arrows being the morphisms that commute with the morphisms to $S$.

\footnotetext{
${ }^{1} \mathrm{~A}$ motivic Haar measure is constructed in the recent paper [4], but uniqueness is still lacking.
} 
We denote by $K_{0}\left(\operatorname{Var}_{S}\right)$ the Grothendieck group of $S$-varieties. It is an abelian group generated by symbols $[X]$, for $X$ an $S$-variety, with the relations $[X]=[Y]$ if $X$ and $Y$ are isomorphic in $\operatorname{Var}_{S}$, and $[X]=[Y]+$ $[X \backslash Y]$ if $Y$ is Zariski closed in $X$. There is a natural ring structure on $K_{0}\left(\operatorname{Var}_{S}\right)$, the product of $[X]$ and $[Y]$ being equal to $\left[X \times{ }_{S} Y\right]$ (here $X \times{ }_{S} Y$ is considered with its reduced structure). Sometimes we will also write $[X / S]$ instead of $[X]$, to emphasize the role of $S$. We write $\mathbf{L}$ to denote the class of $\mathbf{A}_{k}^{1} \times S$ in $K_{0}\left(\operatorname{Var}_{S}\right)$, where the morphism from $\mathbf{A}_{k}^{1} \times S$ to $S$ is the natural projection. We denote by $\mathcal{M}_{S}$ the ring obtained from $K_{0}\left(\operatorname{Var}_{S}\right)$ by inverting $\mathbf{L}$. When $A$ is a constructible subset of some $S$-variety, we define $[A / S]$ in the obvious way, writing $A$ as a disjoint union of a finite number of locally closed subvarieties $A_{i}$. Indeed $[A / S]:=\sum_{i}\left[A_{i} / S\right]$ does not depend on the choice of the subvarieties $A_{i}$.

When $S=\operatorname{Spec}(k)$, we will write $K_{0}\left(\operatorname{Var}_{k}\right)$ instead of $K_{0}\left(\operatorname{Var}_{S}\right)$ and $\mathcal{M}_{k}$ instead of $\mathcal{M}_{S}$.

\subsection{Equivariant Grothendieck groups}

We need some technical preparation in order to take care of the monodromy actions in the next section.

For any positive integer $n$, let $\mu_{n}$ be the group of all $n$-th roots of unity (in some fixed algebraic closure of $k$ ). Note that $\mu_{n}$ is actually an algebraic variety over $k$, namely $\operatorname{Spec}\left(k[x] /\left(x^{n}-1\right)\right)$. The $\mu_{n}$ 's form a projective system, with respect to the maps $\mu_{n d} \rightarrow \mu_{n}: x \mapsto x^{d}$. We denote by $\hat{\mu}$ the projective limit of the $\mu_{n}$. Note that the group $\hat{\mu}$ is not an algebraic variety, but a pro-variety.

Let $X$ be an $S$-variety. A good $\mu_{n}$-action on $X$ is a group action $\mu_{n} \times X \rightarrow X$ which is a morphism of $S$-varieties, such that each orbit is contained in an affine subvariety of $X$. This last condition is automatically satisfied when $X$ is a quasi projective variety. A good $\hat{\mu}$-action on $X$ is an action of $\hat{\mu}$ on $X$ which factors through a good $\mu_{n}$-action, for some $n$.

The monodromic Grothendieck group $K_{0}^{\hat{\mu}}\left(\operatorname{Var}_{S}\right)$ is defined as the abelian group generated by symbols $[X, \hat{\mu}]$ (also denoted by $[X / S, \hat{\mu}]$, or simply $[X])$, for $X$ an $S$-variety with good $\hat{\mu}$-action, with the relations $[X, \hat{\mu}]=[Y, \hat{\mu}]$ if $X$ and $Y$ are isomorphic as $S$-varieties with $\hat{\mu}$-action, and $[X, \hat{\mu}]=[Y, \hat{\mu}]+[X \backslash Y, \hat{\mu}]$ if $Y$ is Zariski closed in $X$ with the $\hat{\mu}$-action on $Y$ induced by the one on $X$, and moreover $[X \times V, \hat{\mu}]=\left[X \times \mathbf{A}_{k}^{n}, \hat{\mu}\right]$ where $V$ is the $n$-dimensional affine space over $k$ with any linear $\hat{\mu}$-action, and $\mathbf{A}_{k}^{n}$ is taken with the trivial $\hat{\mu}$-action. There is a natural ring structure 
on $K_{0}^{\hat{\mu}}\left(\operatorname{Var}_{S}\right)$, the product being induced by the fiber product over $S$. We write $\mathbf{L}$ to denote the class in $K_{0}^{\hat{\mu}}\left(\operatorname{Var}_{S}\right)$ of $\mathbf{A}_{k}^{1} \times S$ with the trivial $\hat{\mu}$-action.

We denote by $\mathcal{M}_{S}^{\hat{\mu}}$ the ring obtained from $K_{0}^{\hat{\mu}}\left(\operatorname{Var}_{S}\right)$ by inverting $\mathbf{L}$. When $A$ is a constructible subset of $X$ which is stable under the $\hat{\mu}$-action, then we define $[A, \hat{\mu}]$ in the obvious way. When $S$ consists of only one geometric point, i.e. $S=\operatorname{Spec}(k)$, then we will write $K_{0}^{\hat{\mu}}\left(\operatorname{Var}_{k}\right)$ instead of $K_{0}^{\hat{\mu}}\left(\operatorname{Var}_{S}\right)$.

Note that for any $s \in S(k)$ we have natural ring morphisms Fiber $_{s}$ : $K_{0}^{\hat{\mu}}\left(\operatorname{Var}_{S}\right) \rightarrow K_{0}^{\hat{\mu}}\left(\operatorname{Var}_{k}\right)$ and Fiber $_{s}: \mathcal{M}_{S}^{\hat{\mu}} \rightarrow \mathcal{M}_{k}^{\hat{\mu}}$ given by $[X, \hat{\mu}] \rightarrow\left[X_{s}, \hat{\mu}\right]$, where $X_{s}$ denotes the fiber at $s$ of $X \rightarrow S$.

We shall also consider the canonical morphism of abelian groups $\iota$ : $K_{0}^{\hat{\mu}}\left(\operatorname{Var}_{S}\right) \rightarrow K_{0}^{\hat{\mu}}\left(\operatorname{Var}_{k}\right)$ which to $[X / S, \hat{\mu}]$ assigns $[X, \hat{\mu}]$. It is in general not a ring morphism, but it can nevertheless be extended to a group morphism $\iota: \mathcal{M}_{S}^{\hat{\mu}} \rightarrow \mathcal{M}_{k}^{\hat{\mu}}$ by sending $\mathbf{L}^{-1}$ to $\mathbf{L}^{-1}[S, \hat{\mu}]$. One should note that $\iota(X \mathbf{L})=$ $\iota(X) \mathbf{L}$ and $\iota\left(X \mathbf{L}^{-1}\right)=\iota(X) \mathbf{L}^{-1}$ for $X$ in $\mathcal{M}_{S}^{\hat{\mu}}$.

\subsection{The arc space of $X$}

Let $k$ be a field of characteristic zero and let $X$ be a variety over $k$. For each natural number $n$ we consider the space $\mathcal{L}_{n}(X)$ of arcs modulo $t^{n+1}$ on $X$. This is an algebraic variety over $k$, whose $K$-rational points, for any field $K$ containing $k$, are the $K[t] / t^{n+1} K[t]$-rational points of $X$. For example when $X$ is an affine variety with equations $f_{i}(x)=0, i=$ $1, \ldots, m, x=\left(x_{1}, \ldots, x_{n}\right)$, then $\mathcal{L}_{n}(X)$ is given by the equations, in the variables $a_{0}, \ldots, a_{n}$, expressing that $f_{i}\left(a_{0}+a_{1} t+\cdots+a_{n} t^{n}\right) \equiv 0 \bmod t^{n+1}$, $i=1, \ldots, m$.

Taking the projective limit of these algebraic varieties $\mathcal{L}_{n}(X)$ we obtain the arc space $\mathcal{L}(X)$ of $X$, which is a reduced separated scheme over $k$. In general, $\mathcal{L}(X)$ is not of finite type over $k($ i.e. $\mathcal{L}(X)$ is an "algebraic variety of infinite dimension"). The $K$-rational points of $\mathcal{L}(X)$ are the $K[[t]]$-rational points of $X$. These are called $K$-arcs on $X$. For example when $X$ is an affine complex variety with equations $f_{i}(x)=0, i=1, \ldots, m, x=\left(x_{1}, \ldots, x_{n}\right)$, then the C-rational points of $\mathcal{L}(X)$ are the sequences $\left(a_{0}, a_{1}, a_{2}, \ldots\right) \in$ $\left(\mathbf{C}^{n}\right)^{\mathbf{N}}$ satisfying $f_{i}\left(a_{0}+a_{1} t+a_{2} t^{2}+\cdots\right)=0$, for $i=1, \ldots, m$. For any $n$, and for $m>n$, we have natural morphisms

$$
\pi_{n}: \mathcal{L}(X) \longrightarrow \mathcal{L}_{n}(X) \text { and } \pi_{n}^{m}: \mathcal{L}_{m}(X) \longrightarrow \mathcal{L}_{n}(X),
$$

obtained by truncation. Note that $\mathcal{L}_{0}(X)=X$ and that $\mathcal{L}_{1}(X)$ is the tangent bundle of $X$. For any arc $\gamma$ on $X$ (i.e. a $K$-arc for some field $K$ 
containing $k$ ), we call $\pi_{0}(\gamma)$ the origin of the arc $\gamma$.

\section{§3. Motivic zeta function}

3.1. Let $k$ be a field of characteristic zero and let $X$ be a smooth variety over $k$. We set $X_{0}:=f^{-1}(0)$. Let $n \geq 1$ be an integer. The morphism $f: X \rightarrow \mathbf{A}_{k}^{1}$ induces a morphism $f: \mathcal{L}_{n}(X) \rightarrow \mathcal{L}_{n}\left(\mathbf{A}_{k}^{1}\right)$.

Any point $\alpha$ of $\mathcal{L}\left(\mathbf{A}_{k}^{1}\right)$, resp. $\mathcal{L}_{n}\left(\mathbf{A}_{k}^{1}\right)$, yields a $K$-rational point, for some field $K$ containing $k$, and hence a power series $\alpha(t) \in K[[t]]$, resp. $\alpha(t) \in K[[t]] / t^{n+1}$. This yields maps

$$
\operatorname{ord}_{t}: \mathcal{L}\left(\mathbf{A}_{k}^{1}\right) \longrightarrow \mathbf{N} \cup\{\infty\}, \quad \operatorname{ord}_{t}: \mathcal{L}_{n}\left(\mathbf{A}_{k}^{1}\right) \longrightarrow\{0,1, \ldots, n, \infty\},
$$

with $\operatorname{ord}_{t} \alpha$ the largest $e$ such that $t^{e}$ divides $\alpha(t)$.

We set

$$
\mathcal{X}_{n}:=\left\{\varphi \in \mathcal{L}_{n}(X) \mid \operatorname{ord}_{t} f(\varphi)=n\right\} .
$$

This is a locally closed subvariety of $\mathcal{L}_{n}(X)$. Note that $\mathcal{X}_{n}$ is actually an $X_{0}$-variety, through the morphism $\pi_{0}^{n}: \mathcal{L}_{n}(X) \rightarrow X$. Indeed $\pi_{0}^{n}\left(\mathcal{X}_{n}\right) \subset X_{0}$, since $n \geq 1$. We consider the morphism

$$
\bar{f}: \mathcal{X}_{n} \longrightarrow \mathbf{G}_{m, k}:=\mathbf{A}_{k}^{1} \backslash\{0\},
$$

sending a point $\varphi$ in $\mathcal{X}_{n}$ to the coefficient of $t^{n}$ in $f(\varphi)$. There is a natural action of $\mathbf{G}_{m, k}$ on $\mathcal{X}_{n}$ given by $a \cdot \varphi(t)=\varphi(a t)$, where $\varphi(t)$ is the vector of power series corresponding to $\varphi$ (in some local coordinate system). Since $\bar{f}(a \cdot \varphi)=a^{n} \bar{f}(\varphi)$ it follows that $\bar{f}$ is a locally trivial fibration for the étale topology.

We denote by $\mathcal{X}_{n}^{1}$ the fiber $\bar{f}^{-1}(1)$. Note that the action of $\mathbf{G}_{m, k}$ on $\mathcal{X}_{n}$ induces a good action of $\mu_{n}$ (and hence of $\hat{\mu}$ ) on $\mathcal{X}_{n}^{1}$. In fact, the $X_{0}$-variety $\mathcal{X}_{n}^{1}$ and the action of $\mu_{n}$ on it completely determines both the variety $\mathcal{X}_{n}$ and the morphism

$$
\left(\bar{f}, \pi_{0}^{n}\right): \mathcal{X}_{n} \longrightarrow \mathbf{G}_{m, k} \times X_{0} .
$$

Indeed it is easy to verify that $\mathcal{X}_{n}$, as a $\left(\mathbf{G}_{m, k} \times X_{0}\right)$-variety, is isomorphic to $\mathcal{X}_{n}^{1} \times{ }^{\mu_{n}} \mathbf{G}_{m, k}$, the quotient of $\mathcal{X}_{n}^{1} \times \mathbf{G}_{m, k}$ under the $\mu_{n}$-action defined by $a(\varphi, b)=\left(a \varphi, a^{-1} b\right)$.

The motivic zeta function of $f: X \rightarrow \mathbf{A}_{k}^{1}$, is the power series over $\mathcal{M}_{X_{0}}^{\hat{\mu}}$ defined by

$$
Z_{f}(T):=\sum_{n \geq 1}\left[\mathcal{X}_{n}^{1} / X_{0}, \hat{\mu}\right] \mathbf{L}^{-n r} T^{n},
$$


with $r$ the dimension of $X$. It is a rational function of $T$ (cf. [1], [2]). When no confusion occurs we shall also write $Z_{f}(T)$ for $\iota\left(Z_{f}(T)\right)$.

For $x$ a closed point of $X_{0}$, one defines similarly $\mathcal{X}_{n, x}$ and $\mathcal{X}_{n, x}^{1}$, by requiring the arcs to have their origin in $x$.

The local motivic zeta function of $f$ at $x$ is the power series in $\mathcal{M}_{x}^{\hat{\mu}}$ defined by

$$
Z_{f, x}(T):=\sum_{n \geq 1}\left[\mathcal{X}_{n, x}^{1}, \hat{\mu}\right] \mathbf{L}^{-n r} T^{n} .
$$

Lemma 3.2. Assume $X$ is the affine space $\mathbf{A}_{k}^{r}$ and $f$ is a homogeneous polynomial of degree $d$ on $\mathbf{A}_{k}^{r}$. Then the equality

$$
Z_{f}(T)=\mathbf{L}^{r} T^{-d} Z_{f, 0}(T)
$$

holds in $\mathcal{M}_{k}^{\hat{\mu}}[[T]]$.

Proof. By homogeneity, the mapping $\varphi \mapsto t \varphi$ induces an isomorphism between $\mathcal{X}_{n}$ and $\pi_{n+1}^{n+d}\left(\mathcal{X}_{n+d, 0}\right)$ compatible with the fibrations $\mathcal{X}_{n} \rightarrow \mathbf{G}_{m, k}$ and $\mathcal{X}_{n+r, 0} \rightarrow \mathbf{G}_{m, k}$. Hence $\left[\mathcal{X}_{n}^{1}, \hat{\mu}\right]=\left[\mathcal{X}_{n+d, 0}^{1}, \hat{\mu}\right] \mathbf{L}^{-(d-1) r}$ and the result follows.

\section{$\S 4$. Statement of the main result}

4.1. Assume $k$ is a field of characteristic zero. Let $G$ be a connected reductive $k$-algebraic group and $\rho$ be a linear representation of $G$ on $\mathbf{A}_{k}^{m}$. Write $m=r_{1}+r_{2}$. Then set $X_{1}=M_{m, r_{1}}$ and let $G \times \mathrm{SL}_{r_{1}}$ act on $X_{1}$ by

$$
\rho_{1}\left(g, g_{1}\right) x_{1}=\rho(g) x_{1}^{t} g_{1} \text {. }
$$

Similarly set $X_{2}=M_{m, r_{2}}$ and let $G \times \mathrm{SL}_{r_{2}}$ act on $X_{2}$ by

$$
\rho_{2}\left(g, g_{2}\right) x_{2}={ }^{t} \rho(g)^{-1} x_{2}{ }^{t} g_{2} .
$$

Assume $G_{j}=\operatorname{Im} \rho_{j}$ is an irreducible regular prehomogeneous vector space for $j=1,2$ with singular locus the irreducible hypersurface $f_{j}=0$. One considers the quotient spaces $X_{i} / \mathrm{SL}_{r_{i}}$ as embedded by the Plücker embedding

$$
X_{i} / \mathrm{SL}_{r_{i}} \longleftrightarrow V_{i}:=\mathbf{A}^{\left(\begin{array}{c}
m \\
r_{i}
\end{array}\right)} \text {. }
$$

There is a natural isomorphism $V_{1} \simeq V_{2}$ under which the quotient spaces $X_{1} / \mathrm{SL}_{r_{1}}$ and $X_{2} / \mathrm{SL}_{r_{2}}$ may be naturally identified. Hence we write $V$ for 
both $V_{1}$ and $V_{2}$, and up to multiplying one of them by a nonzero constant factor, one may assume that both $f_{1}$ and $f_{2}$ are the pullback of the same homogeneous polynomial $f$ of degree $d$ in $V$, cf. [17]. In particular, $\operatorname{deg} f_{j}=$ $r_{j} d$, for $1 \leq j \leq 2$.

THEOREM 4.2. Let $\left(G_{1}, X_{1}\right)$ and $\left(G_{2}, X_{2}\right)$ be irreducible regular prehomogeneous vector spaces which are related by a castling transformation. Then the relations

$$
Z_{f_{1}}(T)\left[\mathrm{SL}_{r_{2}, k}\right] \prod_{1 \leq j \leq r_{1}}\left(1-T^{d} \mathbf{L}^{-j}\right)=Z_{f_{2}}(T)\left[\mathrm{SL}_{r_{1}, k}\right] \prod_{1 \leq j \leq r_{2}}\left(1-T^{d} \mathbf{L}^{-j}\right)
$$

and

$$
\begin{aligned}
Z_{f_{1}, 0}(T) \prod_{1 \leq j \leq r_{1}}\left(T^{-d}-\mathbf{L}^{-j}\right) \prod_{1 \leq j \leq r_{2}}\left(1-\mathbf{L}^{-j}\right) \\
\quad=Z_{f_{2}, 0}(T) \prod_{1 \leq j \leq r_{2}}\left(T^{-d}-\mathbf{L}^{-j}\right) \prod_{1 \leq j \leq r_{1}}\left(1-\mathbf{L}^{-j}\right)
\end{aligned}
$$

hold in $\mathcal{M}_{k}^{\hat{\mu}}[[T]]$, with $\left[\mathrm{SL}_{r}\right]=\mathbf{L}^{r^{2}-1} \prod_{2 \leq i \leq r}\left(1-\mathbf{L}^{-i}\right)$.

\section{§5. Proof of Theorem 4.2}

5.1. Fix $1 \leq r \leq m$ and set $Z=M_{m, r}$. The $k$-group scheme $\mathbf{G L}_{m}(k[[t]])$ acts naturally on $\mathcal{L}(Z)$ on the left, while the $k$-group scheme $\mathrm{SL}_{r}(k[[t]])$ acts naturally on $\mathcal{L}(Z)$ on the right. Here the group of $K$-points of $\mathbf{G L}_{m}(k[[t]])$, resp. $\mathrm{SL}_{r}(k[[t]])$, is $\mathrm{GL}_{m}(K[[t]])$, resp. $\mathrm{SL}_{r}(K[[t]])$, for $K$ a field containing $k$. We shall consider the quotient space $h: Z \rightarrow Y:=$ $Z / \mathrm{SL}_{r}$ as embedded by the Plücker embedding

$$
Z / \mathrm{SL}_{r} \longleftrightarrow V:=\mathbf{A}^{\left(\begin{array}{c}
m \\
r
\end{array}\right)}
$$

We set $\mathcal{Z}:=\mathcal{L}(Z)$ and $\mathcal{Y}:=\mathcal{L}(Y)$. We also denote by $\mathcal{Z}^{\prime}$ the subset of $\mathcal{Z}$ consisting of matrices with a non zero minor of order $r$.

5.2. For every sequence $\underline{e}:=\left(e_{1}, \ldots, e_{r}\right)$ in $\mathbf{N}^{r}$, we denote by $\mathcal{Z}_{\underline{e}}$ the subset of $\mathcal{Z}$ consisting of matrices of the form $M A$ with $M$ in $\mathrm{GL}_{m}(K[[t]])$, and $A$ an upper triangular $(m, r)$ matrix with $t^{e_{1}}, \ldots, t^{e_{r}}$ on the diagonal and coefficients in $K[[t]]$, for some field $K$ containing $k$. The set $\mathcal{Z}^{\prime}$ is the disjoint union of the subsets $\mathcal{Z}_{\underline{e}}$, for $\underline{e}$ in $\mathbf{N}^{r}$. 
5.3. We shall consider the set $\mathcal{V}_{\underline{e}}$ of matrices in $\mathcal{Z}_{\underline{e}}$ with principal $r$ minor (i.e. the one obtained by keeping the first $r$ lines and columns) of valuation $|\underline{e}|:=\sum_{1 \leq i \leq r} e_{i}$. We may describe $\mathcal{V}_{\underline{e}}$ as follows. For $w$ in $W:=\mathcal{S}_{r}$, the permutation group on $r$ letters, we consider the set $\mathcal{V}_{\underline{e}, w}$ of matrices $x=\left(x_{i, j}\right)_{1 \leq i \leq m, 1 \leq j \leq r}$ in $\mathcal{Z}^{\prime}$ of the form

$$
x_{i, j}=a_{i, j} u_{j} t^{e_{j}}+\sum_{1 \leq k<j} \lambda_{k, j} a_{i, k}
$$

with

(a) $\lambda_{k, j}$ and $a_{i, k}$ in $K[[t]]$

(b) $u_{j}$ a unit in $K[[t]]$

(c) $v\left(a_{i, j}\right) \geq 1$ when $i<w(j)$

(d) $a_{w(j), j}=1$

(e) $a_{w(k), j}=0$ when $k<j$.

The set $\mathcal{V}_{\underline{e}}$ is the disjoint union of the subsets $\mathcal{V}_{\underline{e}, w}$. We denote by $\mathcal{W}_{\underline{e}}$ the image of $\overline{\mathcal{V}}_{\underline{e}}$ in $\mathcal{Y}$. Let us remark that $\mathcal{Z}_{\underline{e}}$, resp. $h\left(\mathcal{Z}_{\underline{e}}\right)$, is the union of left translates of $\mathcal{V}_{\underline{e}}$, resp. $\mathcal{W}_{\underline{e}}$, by permutation matrices.

LemmA 5.4. For $n \geq|\underline{e}|$, with $|\underline{e}|:=\sum_{1 \leq i \leq r} e_{i}$, the canonical morphism

$$
\pi_{n}\left(\mathcal{V}_{\underline{e}, w}\right) \longrightarrow \pi_{n}\left(\mathcal{W}_{\underline{e}}\right)
$$

is a locally trivial fibration for the Zariski topology, with fiber $Z_{w} \times \mathbf{A}_{k}^{N}$, where

$$
Z_{w}:=\left(\mathbf{G}_{m, k}\right)^{r-1} \prod_{1 \leq i \leq r} \mathbf{A}_{k}^{r-1-m_{i}},
$$

with $m_{i}$ the number of integers $1 \leq k<w(i)$ such that $k \neq w(j)$ for $j<i$ and

$$
N:=n\left(r^{2}-1\right)+|\underline{e}|((m-r) r+1)-\sum_{1 \leq i \leq r}(m+1-i) e_{i} .
$$

Proof. Let us first consider the case where $w=$ id. Let $x$ be an $(m, r)$ matrix in $\mathcal{V}_{\underline{e} \text {,id. }}$. For $r+1 \leq k \leq m$ and $j \leq r$, we shall denote by $\Delta^{k, j}$ the determinant of the $(r, r)$ submatrix of $x$ obtained by removing the $j$ th line from the $(r+1, r)$ submatrix of $x$ obtained by keeping the first $r$ lines together with the $k$-th line. Set $\Delta:=\prod_{1 \leq i \leq r} u_{i} t^{e_{i}}$. We can take the functions $\Delta^{k, j}$, for $r+1 \leq k \leq m$ and $1 \leq j \leq r$, together with $\Delta$, as coordinates on $\mathcal{W}_{\underline{e}}$. 
We have the relations

$$
\begin{gathered}
\Delta^{k, r}=a_{k, r} \Delta \\
\Delta^{k, r-1}=a_{r, r-1} \Delta^{k, r}-a_{k, r-1} \Delta \\
\ldots \\
\Delta^{k, i}=\sum_{1 \leq j \leq r-i}(-1)^{j+1} a_{i+j, i} \Delta^{k, i+j}+(-1)^{r-i} a_{k, i} \Delta \\
\cdots \\
\Delta^{k, 1}=a_{2,1} \Delta^{k, 2}-a_{3,1} \Delta^{k, 3}+\cdots+(-1)^{r} a_{r, 1} \Delta^{k, r}+(-1)^{r+1} a_{k, 1} \Delta .
\end{gathered}
$$

Let us remark that it follows that the set $\mathcal{W}_{\underline{e}}$ is the set defined by the conditions $\operatorname{ord}_{t}(\Delta)=|\underline{e}|$ and $\operatorname{ord}_{t}\left(\Delta^{k, j}\right) \geq|\underline{e}|$, for $r+1 \leq k \leq m$ and $1 \leq j \leq r$.

Let us assume $n \geq|\underline{e}|$. For a fixed point $y$ in $\mathcal{W}_{\underline{e}}$ with coordinates $\left(\delta t^{|\underline{e}|}, \delta^{k, j} \delta t^{|\underline{e}|}\right)$ (hence $\delta$ is a unit and $\operatorname{ord}_{t}\left(\delta^{k, j}\right) \geq 0$ ) the conditions $\pi_{n}(h(x))=\pi_{n}(y)$ may be rewritten as

$$
\prod_{1 \leq i \leq r} u_{i} \equiv \delta \quad \bmod t^{n+1-|\underline{e}|}
$$

$$
a_{k, r} \equiv \delta^{k, r} \bmod t^{n+1-|\underline{e}|}
$$

$\left(*_{r-1}\right)$

$$
a_{k, r-1} \equiv a_{r, r-1} \delta^{k, r}-\delta^{k, r-1} \bmod t^{n+1-|\underline{e}|}
$$

$$
\begin{gathered}
a_{k, i} \equiv \sum_{1 \leq j \leq r-i}(-1)^{r-i-j} a_{i+j, i} \delta^{k, i+j}+(-1)^{r-i} \delta^{k, i} \bmod t^{n+1-|\underline{e}|} \\
\cdots \\
a_{k, 1} \equiv a_{r, 1} \delta^{k, r}-a_{r-1,1} \delta^{k, r-1}+\cdots \\
\cdots+(-1)^{r} a_{2,1} \delta^{k, 2}+(-1)^{r-1} \delta^{k, 1} \bmod t^{n+1-|\underline{e}|}
\end{gathered}
$$

It follows from that description that the morphism $\pi_{n}\left(\mathcal{V}_{\underline{e}, w}\right) \rightarrow \pi_{n}\left(\mathcal{W}_{\underline{e}}\right)$ is a locally trivial fibration (in fact, a trivial fibration) for the Zariski topology and that the fiber is of the form $\mathbf{G}_{m, k}^{d-1} \times \mathbf{A}_{k}^{N^{\prime}}$, for some integer $N^{\prime}$. Indeed, if we expand relation $(*)$ in terms of the coefficients $u_{i, j}$ and $\delta_{j}$ of the power series $u_{i}$ and $\delta$, one gets the equality $\prod_{1 \leq i \leq r} u_{i, 0}=\delta_{0}$, with $u_{i, 0} \neq$ 0 , which gives rise to the factor $\mathbf{G}_{m, k}^{d-1}$, and some other equations which at each level are linear with non zero coefficients in the newly occuring variables. On the other hand, once expanded, the relations $\left(*_{r}\right), \ldots,\left(*_{1}\right)$ 
give a triangular system of linear equations in the power series coefficients of the variables $a_{i, j}$. This gives the result except for the exact value of $N^{\prime}$ which can be obtained either by direct calculation or by computing $\operatorname{dim} \pi_{n}\left(\mathcal{V}_{\underline{e}, w}\right)-\operatorname{dim} \pi_{n}\left(\mathcal{W}_{\underline{e}}\right)$.

For arbitrary $w$, the situation reduces to the former one, since, up to renumbering the rows, the situation is just the same except that some $a_{i, j}$ 's with $i, j \leq r$ are now required to be of valuation $\geq 1$, which has the sole effect of replacing $Z_{\text {id }}$ by $Z_{w}$ in the statement.

Lemma 5.5. Set $\mathcal{Y}_{0}:=h\left(\mathcal{Z}_{\underline{0}}\right)$ and, for $k \geq 0, \mathcal{Y}_{k}=t^{k} \mathcal{Y}_{0}$.

(1) For every $\underline{e}$ in $\mathbf{N}^{r}, h\left(\mathcal{Z}_{\underline{e}}\right)=\mathcal{Y}_{|\underline{e}|}$.

(2) Assume $n \geq k \geq 0$. For every constructible subset $A$ of $\pi_{n}\left(\mathcal{Y}_{k}\right)$, consider the preimage $\left[h^{-1}(A)\right]$ of $A$ in $\pi_{n}(\mathcal{Z})$. The relation

$$
\left[h^{-1}(A)\right]=[A]\left[\mathrm{SL}_{r}\right] \mathbf{L}^{n\left(r^{2}-1\right)+k((m-r) r+1)} \sum_{|\underline{e}|=k} \prod_{1 \leq i \leq r} \mathbf{L}^{-(m+1-i) e_{i}}
$$

holds in $\mathcal{M}_{k}$. Furthermore, if $A$ is constructible with $\hat{\mu}$-action, the same relation still holds in $\mathcal{M}_{k}^{\hat{\mu}}$.

Proof. Assertion (1) follows from the fact, noted in the proof of Lemma 5.4, that the set $\mathcal{W}_{\underline{e}}$ depends only on $|\underline{e}|$. Let us denote it by $\mathcal{W}_{|\underline{e}|}$.

To prove (2), we may assume $A$ is contained in $\pi_{n}\left(\mathcal{W}_{k}\right)$. Since the preimage of $\pi_{n}\left(\mathcal{W}_{k}\right)$ in $\pi_{n}(\mathcal{Z})$ is the disjoint union of the sets $\pi_{n}\left(\mathcal{V}_{\underline{e}, w}\right)$ with $|\underline{e}|=k$ and $w$ in $W$, we have

$$
\left[h^{-1}(A)\right]=\sum_{|\underline{e}|=k}\left[h^{-1}(A) \cap \pi_{n}\left(\mathcal{V}_{\underline{e}, w}\right)\right] .
$$

One the other hand, by Lemma 5.4, we have

$$
\left[h^{-1}(A) \cap \pi_{n}\left(\mathcal{V}_{\underline{e}, w}\right)\right]=\left[Z_{w}\right] \times \mathbf{L}^{n\left(r^{2}-1\right)+|\underline{e}|((m-r) r+1)-\sum_{1 \leq i \leq r}(m+1-i) e_{i}},
$$

hence the result follows now from the relation

$$
\sum_{w \in W}\left[Z_{w}\right]=\mathbf{L}^{r^{2}-1} \prod_{2 \leq i \leq r}\left(1-\mathbf{L}^{-i}\right)=\left[\mathrm{SL}_{r}\right]
$$


5.6. Let $f$ be a homogeneous polynomial of degree $d$ on $V$. We set

$$
\mathcal{Y}_{n, k}:=\left\{\varphi \in \mathcal{L}_{n}(Y) \mid \varphi \in \pi_{n}\left(\mathcal{Y}_{k}\right) \text { and } \operatorname{ord}_{t} f(\varphi)=n\right\}
$$

As in 3.1, we define $\bar{f}: \mathcal{Y}_{n, k} \rightarrow \mathbf{G}_{m, k}$, and we set $\mathcal{Y}_{n, k}^{1}:=\bar{f}^{-1}(1)$, which is a variety with $\hat{\mu}$-action. We shall also consider the varieties $\mathcal{X}_{n, k}$ and $\mathcal{X}_{n, k}^{1}$ which are the inverse images of respectively $\mathcal{Y}_{n, k}$ and $\mathcal{Y}_{n, k}^{1}$ in $\mathcal{L}_{n}(Z)$.

LEMMA 5.7. The relation

$$
\left[\mathcal{Y}_{n, k}^{1}\right]=\left[\mathcal{Y}_{n-k d, 0}^{1}\right] \mathbf{L}^{k(d-1) \operatorname{dim} Y}
$$

holds in $\mathcal{M}_{k}^{\hat{\mu}}$.

Proof. By homogeneity, the mapping $y \mapsto t^{k} y$ induces an isomorphism between $\mathcal{Y}_{n-k d, 0}$ and $\pi_{n-k(d-1)}^{n}\left(\mathcal{Y}_{n, k}\right)$ compatible with the fibrations onto $\mathbf{G}_{m, k}$.

5.8. Let us now consider the motivic zeta function of $f \circ h$ (or more precisely its image in $\left.\mathcal{M}_{k}^{\hat{\mu}}[[T]]\right)$,

$$
Z_{f \circ h}(T):=\sum_{n \geq 1}\left[\mathcal{X}_{n}^{1}, \hat{\mu}\right] \mathbf{L}^{-n \operatorname{dim} Z} T^{n}
$$

where $\mathcal{X}_{n}^{1}$ is the disjoint union of the varieties with $\hat{\mu}$-action $\mathcal{X}_{n, k}^{1}, k$ in $\mathbf{N}$. Remark that for $k d>n, \mathcal{X}_{n, k}$ and $\mathcal{Y}_{n, k}$ are empty, hence for fixed $n$ only a finite number of the $\mathcal{X}_{n, k}^{1}$ 's are non empty. We shall also consider the power series

$$
Z_{f \circ h}^{0}(T):=\sum_{n \geq 1}\left[\mathcal{X}_{n, 0}^{1}, \hat{\mu}\right] \mathbf{L}^{-n \operatorname{dim} Z} T^{n}
$$

and

$$
Z_{f}^{0}(T):=\sum_{n \geq 1}\left[\mathcal{Y}_{n, 0}^{1}, \hat{\mu}\right] \mathbf{L}^{-n \operatorname{dim} Y} T^{n}
$$

in $\mathcal{M}_{k}^{\hat{\mu}}[[T]]$.

Proposition 5.9. The relations

$$
Z_{f \circ h}(T)=\prod_{1 \leq i \leq r}\left(1-\mathbf{L}^{-(m+1-i)} T^{d}\right)^{-1} Z_{f \circ h}^{0}(T)
$$


and

$$
Z_{f \circ h}(T)=\left[\mathrm{SL}_{r}\right] \prod_{1 \leq i \leq r}\left(1-\mathbf{L}^{-(m+1-i)} T^{d}\right)^{-1} Z_{f}^{0}(T)
$$

hold in $\mathcal{M}_{k}^{\hat{\mu}}[[T]]$.

Proof. Since, as follows from Lemma 5.5,

$$
Z_{f \circ h}^{0}(T)=\left[\mathrm{SL}_{r}\right] Z_{f}^{0}(T)
$$

it is enough to prove (5.9.2). By Lemma 5.5, we have

$$
\left[\mathcal{X}_{n, k}^{1}\right] \mathbf{L}^{-n \operatorname{dim} Z}=\left[\mathrm{SL}_{r}\right]\left[\mathcal{Y}_{n, k}^{1}\right] \mathbf{L}^{-n \operatorname{dim} Y} \mathbf{L}^{k \operatorname{dim} Y} \sum_{|\underline{e}|=k} \prod_{1 \leq i \leq r} \mathbf{L}^{-(m+1-i) e_{i}}
$$

It follows from Lemma 5.7 that we may rewrite the last equality as

$$
\left[\mathcal{X}_{n, k}^{1}\right] \mathbf{L}^{-n \operatorname{dim} Z}=\left[\mathrm{SL}_{r}\right]\left[\mathcal{Y}_{n-k d, 0}^{1}\right] \mathbf{L}^{-(n-k d) \operatorname{dim} Y} \sum_{|\underline{e}|=k} \prod_{1 \leq i \leq r} \mathbf{L}^{-(m+1-i) e_{i}}
$$

and we get the result by summing up the series.

5.10. Now we can conclude the proof of Theorem 4.2. Relation (4.2.1) follows from writing (5.9.2) for both $X_{1}$ and $X_{2}$, and (4.2.2) follows from (4.2.1) together with Lemma 3.2.

\section{§6. Applications to the Milnor fibre and the Hodge spectrum}

\subsection{Monodromy}

In this subsection 6.1 we assume that $k=\mathbf{C}$. Let $x$ be a point of $X_{0}=$ $f^{-1}(0)$. We fix a smooth metric on $X$. We set $X_{\varepsilon, \eta}^{\times}:=B(x, \varepsilon) \cap f^{-1}\left(D_{\eta}^{\times}\right)$, with $B(x, \varepsilon)$ the open ball of radius $\varepsilon$ centered at $x$ and $D_{\eta}^{\times}:=D_{\eta} \backslash\{0\}$, with $D_{\eta}$ the open disk of radius $\eta$ centered at 0 . For $0<\eta \ll \varepsilon \ll 1$, the restriction of $f$ to $X_{\varepsilon, \eta}^{\times}$is a locally trivial fibration, called the Milnor fibration, onto $D_{\eta}^{\times}$with fiber $F_{x}$, the Milnor fiber at $x$. The action of a characteristic homeomorphism of this fibration on cohomology gives rise to the monodromy operator

$$
M_{x}: H^{\cdot}\left(F_{x}, \mathbf{Q}\right) \longrightarrow H^{\cdot}\left(F_{x}, \mathbf{Q}\right)
$$




\subsection{The motivic Milnor fibre}

It is a remarkable fact, proved in [1] and [2], that expanding the rational function $Z_{f}(T)$ as a power series in $T^{-1}$ and taking minus its constant term, yields a well defined element $\mathcal{S}_{f}=-\lim _{T \rightarrow \infty} Z_{f}(T)$ of $\mathcal{M}_{X_{0}}^{\hat{\mu}}$. This follows from a formula for the motivic zeta function in terms of embedded resolutions of $X_{0}$ which we now recall.

Let $(Y, h)$ be a resolution of $f$. By this, we mean that $Y$ is a nonsingular and irreducible algebraic variety over $k, h: Y \rightarrow X$ is a proper morphism, that the restriction $h: Y \backslash h^{-1}\left(X_{0}\right) \rightarrow X \backslash X_{0}$ is an isomorphism, and that $h^{-1}\left(X_{0}\right)$ has only normal crossings as a subvariety of $Y$.

We denote by $E_{i}, i \in J$, the irreducible components (over $k$ ) of $h^{-1}\left(X_{0}\right)$. For each $i \in J$, denote by $N_{i}$ the multiplicity of $E_{i}$ in the divisor of $f \circ h$ on $Y$, and by $\nu_{i}-1$ the multiplicity of $E_{i}$ in the divisor of $h^{*} d x$, where $d x$ is a local non vanishing volume form at any point of $h\left(E_{i}\right)$, i.e. a local generator of the sheaf of differential forms of maximal degree. For $i \in J$ and $I \subset J$, we consider the nonsingular varieties $E_{i}^{\circ}:=E_{i} \backslash \bigcup_{j \neq i} E_{j}, E_{I}=\bigcap_{i \in I} E_{i}$, and $E_{I}^{\circ}:=E_{I} \backslash \bigcup_{j \in J \backslash I} E_{j}$.

Let $m_{I}=\operatorname{gcd}\left(N_{i}\right)_{i \in I}$. We introduce an unramified Galois cover $\widetilde{E}_{I}^{\circ}$ of $E_{I}^{\circ}$, with Galois group $\mu_{m_{I}}$, as follows. Let $U$ be an affine Zariski open subset of $Y$, such that, on $U, f \circ h=u v^{m_{I}}$, with $u$ a unit on $U$ and $v$ a morphism from $U$ to $\mathbf{A}_{k}^{1}$. Then the restriction of $\widetilde{E}_{I}^{\circ}$ above $E_{I}^{\circ} \cap U$, denoted by $\widetilde{E}_{I}^{\circ} \cap U$, is defined as

$$
\left\{(z, y) \in \mathbf{A}_{k}^{1} \times\left(E_{I}^{\circ} \cap U\right) \mid z^{m_{I}}=u^{-1}\right\} .
$$

Note that $E_{I}^{\circ}$ can be covered by such affine open subsets $U$ of $Y$. Gluing together the covers $\widetilde{E}_{I}^{\circ} \cap U$, in the obvious way, we obtain the cover $\widetilde{E}_{I}^{\circ}$ of $E_{I}^{\circ}$ which has a natural $\mu_{m_{I}}$-action (obtained by multiplying the $z$-coordinate with the elements of $\left.\mu_{m_{I}}\right)$. This $\mu_{m_{I}}$-action on $\widetilde{E}_{I}^{\circ}$ induces an $\hat{\mu}$-action on $\widetilde{E}_{I}^{\circ}$ in the obvious way.

TheOREM 6.3. ([3], [13]) With the previous notations, the following relation holds in $\mathcal{M}_{X_{0}}^{\hat{\mu}}[[T]]$ :

$$
Z_{f}(T)=\sum_{\emptyset \neq I \subset J}(\mathbf{L}-1)^{|I|-1}\left[\widetilde{E}_{I}^{\circ} / X_{0}, \hat{\mu}\right] \prod_{i \in I} \frac{\mathbf{L}^{-\nu_{i}} T^{N_{i}}}{1-\mathbf{L}^{-\nu_{i}} T^{N_{i}}} .
$$


Definition 6.3.1. ([1], [3], [2]) Expanding the rational function $Z_{f}(T)$ as a power series in $T^{-1}$ and taking minus its constant term, yields a well defined element of $\mathcal{M}_{X_{0}}^{\hat{\mu}}$, namely

$$
\mathcal{S}_{f}:=-\lim _{T \rightarrow \infty} Z_{f}(T):=\sum_{\emptyset \neq I \subset J}(1-\mathbf{L})^{|I|-1}\left[\widetilde{E}_{I}^{\circ}\right] .
$$

Moreover we set $\mathcal{S}_{f, x}:=\operatorname{Fiber}_{x}\left(\mathcal{S}_{f}\right)$ in $\mathcal{M}_{k}^{\hat{\mu}}$. We also have $\mathcal{S}_{f, x}=-\lim _{T \rightarrow \infty}$ $Z_{f, x}(T)$.

There is strong evidence that $\mathcal{S}_{f, x}$ is the correct virtual motivic incarnation of the Milnor fiber $F_{x}$ of $f$ at $x$ (which is in itself not at all motivic).

In the present setting we deduce from Theorem 4.2, the following relation between the virtual motivic Milnor fibres at the origin of two irreducible regular prehomogeneous vector spaces which are related by a castling transformation.

TheOrem 6.4. Assume $k$ is a field of characteristic zero. Let $\left(G_{1}, X_{1}\right)$ and $\left(G_{2}, X_{2}\right)$ be irreducible regular prehomogeneous vector spaces which are related by a castling transformation. Then the relation

$$
\mathcal{S}_{f_{1}, 0} \prod_{1 \leq j \leq r_{2}}\left(1-\mathbf{L}^{j}\right)=\mathcal{S}_{f_{2}, 0} \prod_{1 \leq j \leq r_{1}}\left(1-\mathbf{L}^{j}\right)
$$

holds in $\mathcal{M}_{k}^{\hat{\mu}}$.

Proof. Follows directly from (4.2.2).

\subsection{Hodge structures}

From now on in the remaining of this section 6 , we shall assume $k=\mathbf{C}$. A Hodge structure is a finite dimensional $\mathbf{Q}$-vector space $H$ together with a bigrading $H \otimes \mathbf{C}=\bigoplus_{p, q \in \mathbf{Z}} H^{p, q}$, such that $H^{q, p}$ is the complex conjugate of $H^{p, q}$ and each weight $m$ summand, $\bigoplus_{p+q=m} H^{p, q}$, is defined over $\mathbf{Q}$. The Hodge structures, with the evident notion of morphism, form an abelian category HS with tensor product. The elements of the Grothendieck group $K_{0}$ (HS) of this abelian category are representable as a formal difference of Hodge structures $[H]-\left[H^{\prime}\right]$, and $[H]=\left[H^{\prime}\right]$ iff $H \cong H^{\prime}$. Note that $K_{0}(\mathrm{HS})$ becomes a ring with respect to the tensor product.

A mixed Hodge structure is a finite dimensional Q-vector space $V$ with a finite increasing filtration $W \bullet V$, called the weight filtration, such that the 
associated graded vector space $\mathrm{Gr}_{\bullet}^{W}(V)$ underlies a Hodge structure having $\operatorname{Gr}_{m}^{W}(V)$ as weight $m$ summand. Note that $V$ determines in a natural way an element $[V]$ in $K_{0}(\mathrm{HS})$, namely $[V]:=\sum_{m}\left[\operatorname{Gr}_{m}^{W}(V)\right]$.

When $X$ is an algebraic variety over $k=\mathbf{C}$, the simplicial cohomology groups $H_{c}^{i}(X, \mathbf{Q})$ of $X$, with compact support, underly a natural mixed Hodge structure, and the Hodge characteristic $\chi_{h}(X)$ of $X$ (with compact support) is defined by

$$
\chi_{h}(X):=\sum_{i}(-1)^{i}\left[H_{c}^{i}(X, \mathbf{Q})\right] \in K_{0}(\mathrm{HS}) .
$$

This yields a map $\chi_{h}: \operatorname{Var}_{\mathbf{C}} \rightarrow K_{0}$ (HS) which factors through $\mathcal{M}_{\mathbf{C}}$, because $\chi_{h}\left(\mathbf{A}_{k}^{1}\right)$ is actually invertible in the ring $K_{0}(\mathrm{HS})$. When $X$ is proper and smooth, the mixed Hodge structure on $H_{c}^{i}(X, \mathbf{Q})$ is in fact a Hodge structure, the weight filtration being concentrated in weight $i$.

\subsection{The Hodge spectrum}

The cohomology groups $H^{i}\left(F_{x}, \mathbf{Q}\right)$ of the Milnor fiber $F_{x}$ carry a natural mixed Hodge structure ([20], [22], [15], [16]), which is compatible with the semi-simplification of the monodromy operator $M_{x}$. Hence we can define the Hodge characteristic $\chi_{h}\left(F_{x}\right)$ of $F_{x}$ by

$$
\chi_{h}\left(F_{x}\right):=\sum_{i}(-1)^{i}\left[H^{i}\left(F_{x}, \mathbf{Q}\right)\right] \in K_{0}(\mathrm{HS}) .
$$

Actually by taking into account the monodromy action we can consider $\chi_{h}\left(F_{x}\right)$ as an element of the Grothendieck group $K_{0}\left(\mathrm{HS}^{\mathrm{mon}}\right)$ of the abelian category $\mathrm{HS}^{\mathrm{mon}}$ of Hodge structures with an endomorphism of finite order. Again $K_{0}\left(\mathrm{HS}^{\mathrm{mon}}\right)$ is a ring by the tensor product.

There is a natural linear map, called the Hodge spectrum

$$
\text { hsp : } K_{0}\left(\mathrm{HS}^{\text {mon }}\right) \longrightarrow \mathbf{Z}\left[t^{1 / \mathbf{Z}}\right]:=\bigcup_{n \geq 1} \mathbf{Z}\left[t^{1 / n}, t^{-1 / n}\right],
$$

with $\operatorname{hsp}([H]):=\sum_{\alpha \in \mathbf{Q} \cap[0,1[} t^{\alpha}\left(\sum_{p, q \in \mathbf{Z}} \operatorname{dim}\left(H^{p, q}\right)_{\alpha}\right) t^{p}$, for any Hodge structure $H$ with an endomorphism of finite order, where $H_{\alpha}^{p, q}$ is the generalized eigenspace of $H^{p, q}$ with respect to the eigenvalue $e^{2 \pi \sqrt{-1} \alpha}$.

Let us denote by $r$ the dimension of $X$. We recall that $\operatorname{hsp}(f, x):=$ $(-1)^{r-1} \operatorname{hsp}\left(\chi_{h}\left(F_{x}\right)-1\right)$ is called the Hodge spectrum of $f$ at $x$.

We denote by $\chi_{h}$ the canonical ring homomorphism (called the Hodge characteristic)

$$
\chi_{h}: \mathcal{M}_{\mathbf{C}}^{\hat{\mu}} \longrightarrow K_{0}\left(\mathrm{HS}^{\mathrm{mon}}\right)
$$


which associates to any complex algebraic variety $Z$, with a good $\mu_{n}$-action, its Hodge characteristic together with the endomorphism induced by $Z \rightarrow$ $Z: z \mapsto e^{2 \pi \sqrt{-1} / n} z$.

TheOrem 6.7. ([1]) Assume the above notation with $k=\mathbf{C}$. Then we have the following equality in $K_{0}\left(\mathrm{HS}^{\mathrm{mon}}\right)$ :

$$
\chi_{h}\left(F_{x}\right)=\chi_{h}\left(\mathcal{S}_{f, x}\right)
$$

In particular it follows that

$$
\operatorname{hsp}(f, x):=(-1)^{r-1} \operatorname{hsp}\left(\chi_{h}\left(\mathcal{S}_{f, x}\right)-1\right),
$$

thus the motivic zeta function $Z_{f, x}(T)$ completely determines the Hodge spectrum of $f$ at $x$.

Hence we deduce from Theorem 6.4, since $\operatorname{hsp}\left(\chi_{h}(\mathbf{L})\right)=t$, the following:

Corollary 6.8. Assume $k=$ C. Let $\left(G_{1}, X_{1}\right)$ and $\left(G_{2}, X_{2}\right)$ be irreducible regular prehomogeneous vector spaces which are related by a castling transformation. Then the following relation holds between the Hodge spectra of $f_{1}$ and $f_{2}$ at 0

$$
\frac{1+(-1)^{m r_{1}-1} \operatorname{hsp}\left(f_{1}, 0\right)}{\prod_{1 \leq j \leq r_{1}}\left(1-t^{j}\right)}=\frac{1+(-1)^{m r_{2}-1} \operatorname{hsp}\left(f_{2}, 0\right)}{\prod_{1 \leq j \leq r_{2}}\left(1-t^{j}\right)} .
$$

\section{§7. Some generalizations}

7.1. Assume $k$ is a field of characteristic zero. We now consider the general case of prehomogeneous vector spaces which are not necessarily irreducible and regular. In this setting we are given a connected linear algebraic group $G$ together with a linear action $\rho: G \rightarrow$ End $X$ on a finite dimensional (linear) affine space $X$ over $k$, such that the action is transitive on a dense open set $O=X \backslash S$. Let $S_{j}, 1 \leq j \leq \ell$, be the $k$-irreducible components of the singular set $S$ which are of codimension 1 in $X$ and choose for every $j$ a defining equation $f_{j}=0$ of $S_{j}$. Recall that a non-zero $k$-rational function on $X$ is a $k$-relative invariant of the $G$-action on $X$, if there exists a $k$-rational character $\nu$ of $G$ such that

$$
f(\rho(g) x)=\nu(g) f(x)
$$


for every $g$ in $G$ and $x$ in $X$. The functions $f_{j}$ are $k$-relative invariants and furthermore they are a basis of $k$-relative invariants in the sense that any $k$-relative invariant of $(G, \rho)$ is of the form $c f_{1}^{\mu_{1}} \cdots f_{\ell}^{\mu_{\ell}}$ with $c$ in $k^{\times}$and $\mu_{j}$ in $\mathbf{Z}$.

\subsection{Motivic zeta function for several functions}

Let $X$ be a smooth $k$-variety of dimension $r$ and consider $\ell$ functions $f_{j}: X \rightarrow \mathbf{A}_{k}^{1}$. We set $X_{0}:=\bigcap_{1 \leq j \leq \ell}\left(f_{j}=0\right)$.

For every $n=\left(n_{1}, \ldots, n_{\ell}\right)$ in $\left(\mathbf{N}^{\times}\right)^{\ell}$, we set $|n|:=\sum_{1 \leq j \leq \ell} n_{j}$ and define

$$
\mathcal{X}_{n}:=\left\{x \in \mathcal{L}_{|n|}(X) \mid \operatorname{ord}_{t} f_{j}=n_{j}, 1 \leq j \leq \ell\right\} .
$$

Similarly as in 3.1, we have a natural morphism $\bar{f}: \mathcal{X}_{n} \rightarrow \mathbf{G}_{m, k}^{\ell}$, which makes $\mathcal{X}_{n}$ a $X_{0} \times \mathbf{G}_{m, k}^{\ell}$-variety, for $n$ in $\left(\mathbf{N}^{\times}\right)^{\ell}$. The motivic zeta function attached to $f=\left(f_{1}, \ldots, f_{\ell}\right)$ is the formal series

$$
Z_{f}(T):=\sum_{n \in(\mathbf{N} \times)^{\ell}}\left[\mathcal{X}_{n} / X_{0} \times \mathbf{G}_{m, k}^{\ell}\right] \mathbf{L}^{-|n| r} T^{n}
$$

in $\mathcal{M}_{X_{0} \times \mathbf{G}_{m, k}^{\ell}}\left[\left[T_{1}, \ldots, T_{\ell}\right]\right]$. It is a rational function of $T=\left(T_{1}, \ldots, T_{\ell}\right)$ (cf. [1], [13], [2]). When $\ell=1$, the relation with the definition in 3.1 is the following: the zeta function we just defined is the image of the former one by the canonical morphism $\mathcal{M}_{X_{0}}^{\hat{\mu}} \rightarrow \mathcal{M}_{X_{0} \times \mathbf{G}_{m, k}}$ which to the class of a $X_{0^{-}}$ variety $Y$ with $\hat{\mu}$-action assigns the class of $Y \times{ }^{\hat{\mu}} \mathbf{G}_{m, k}$ in $\mathcal{M}_{X_{0} \times \mathbf{G}_{m, k}}$. For $x$ a point in $X_{0}(k)$, one defines similarly as in the $\ell=1$ case, $Z_{f, x}(T)$ in $\mathcal{M}_{\mathbf{G}_{m, k}^{\ell}}\left[\left[T_{1}, \ldots, T_{\ell}\right]\right]$.

7.3. As in 4.1 we consider a connected linear algebraic group $G$ over $k$ and $\rho$ be a representation of $G$ in $\mathbf{A}_{k}^{m}$. Write $m=r_{1}+r_{2}$ and define $X_{j}$, $G_{j}$ and $\rho_{j}, j=1,2$ as in 4.1. We assume they give rise to prehomogeneous vector spaces for $j=1,2$, and we say that the prehomogeneous vector spaces $\left(G_{1}, X_{1}\right)$ and $\left(G_{2}, X_{2}\right)$ are related by a castling transformation. We choose a basis of $k$-relative invariants $f_{1}=\left(f_{1, i}\right), 1 \leq j \leq \ell_{1}$ and $f_{2}=\left(f_{1,2}\right)$, $1 \leq j \leq \ell_{2}$, respectively. As in 4.1, we consider the quotient spaces $X_{i} / \mathrm{SL}_{r_{i}}$ as embedded by the Plücker embedding the same affine space $V$, and up to renumbering the $f_{1, i}$ 's and multiplying them by non-zero constants, one may assume that both the $f_{1, i}$ 's and the $f_{2, i}$ 's are the pullback of the same homogeneous polynomials $f_{i}$ of degree $d_{i}$ in $V$, cf. [17], [6]. In particular, we may write $\ell_{1}=\ell_{2}=\ell$. We set $d=\left(d_{i}\right)$ in $\left(\mathbf{N}^{\times}\right)^{\ell}$.

We have the following generalization of Theorem 4.2. 
TheOREM 7.4. Let $\left(G_{1}, X_{1}\right)$ and $\left(G_{2}, X_{2}\right)$ be prehomogeneous vector spaces related by a castling transformation. Then the relations

$$
Z_{f_{1}}(T)\left[\mathrm{SL}_{r_{2}, k}\right] \prod_{1 \leq j \leq r_{1}}\left(1-T^{d} \mathbf{L}^{-j}\right)=Z_{f_{2}}(T)\left[\mathrm{SL}_{r_{1}, k}\right] \prod_{1 \leq j \leq r_{2}}\left(1-T^{d} \mathbf{L}^{-j}\right)
$$

and

$$
\begin{aligned}
Z_{f_{1}, 0}(T) \prod_{1 \leq j \leq r_{1}}\left(T^{-d}-\mathbf{L}^{-j}\right) & \prod_{1 \leq j \leq r_{2}}\left(1-\mathbf{L}^{-j}\right)= \\
& Z_{f_{2}, 0}(T) \prod_{1 \leq j \leq r_{2}}\left(T^{-d}-\mathbf{L}^{-j}\right) \prod_{1 \leq j \leq r_{1}}\left(1-\mathbf{L}^{-j}\right)
\end{aligned}
$$

hold in $\mathcal{M}_{\mathbf{G}_{m, k}^{\ell}}[[T]]$, with $T=\left(T_{1}, \ldots, T_{\ell}\right)$ and $\left[\mathrm{SL}_{r}\right]=\mathbf{L}^{r^{2}-1} \prod_{2 \leq i \leq r}(1-$ $\left.\mathbf{L}^{-i}\right)$.

Proof. The proof is just the same as the proof of Theorem 4.2.

Remark 7.5. There is an obvious generalization of Theorem 7.4 to parabolic castling transforms as introduced in [19], cf. [6]. Details are left to the reader.

7.6. Similarly as for the case of one function in 6.3.1, Guibert proves in [5] that, for every $\alpha$ in $\left(\mathbf{N}^{\times}\right)^{\ell},-\lim _{T \rightarrow \infty} Z_{f}\left(T^{\alpha}\right)$ is a well defined element of $\mathcal{M}_{X_{0} \times \mathbf{G}_{m, \mathbf{C}}^{\ell}}$, independent of $\alpha$. Let us denote it by $\mathcal{S}_{f}$ and set $\mathcal{S}_{f, x}:=$ $\operatorname{Fiber}_{x}\left(\mathcal{S}_{f}\right)$. We also have $\mathcal{S}_{f, x}=-\lim _{T \rightarrow \infty} Z_{f, x}\left(T^{\alpha}\right)$, for every $\alpha$ in $\left(\mathbf{N}^{\times}\right)^{\ell}$.

Remark 7.7. When $k=\mathbf{C}$, G. Guibert shows in [5] how Sabbah's Alexander invariants of $f$ (cf. [14]) may be recovered from the motivic zeta function $Z_{f}$.

Remark 7.8. It would be interesting to investigate what information is contained in the Hodge realization of $\mathcal{S}_{f}$. It is quite likely that there should be some connections with recent work of A. Libgober in [12].

The following statement follows directly from Theorem 7.4.

TheOREm 7.9. Let $\left(G_{1}, X_{1}\right)$ and $\left(G_{2}, X_{2}\right)$ be prehomogeneous vector spaces related by a castling transformation. Then the relation

$$
\mathcal{S}_{f_{1}, 0} \prod_{1 \leq j \leq r_{2}}\left(1-\mathbf{L}^{j}\right)=\mathcal{S}_{f_{2}, 0} \prod_{1 \leq j \leq r_{1}}\left(1-\mathbf{L}^{j}\right)
$$

holds in $\mathcal{M}_{X_{0} \times \mathbf{G}_{m, \mathbf{C}}^{\ell}}$. 


\section{REFERENCES}

[1] J. Denef and F. Loeser, Motivic Igusa zeta functions, J. Algebraic Geom., 7 (1998), 505-537.

[2] J. Denef and F. Loeser, Geometry on arc spaces of algebraic varieties, Proceedings of 3rd European Congress of Mathematics, Barcelona 2000, Progress in Mathematics 201, Birkhaüser (2001), pp. 327-348.

[3] J. Denef and F. Loeser, Lefschetz numbers of iterates of the monodromy and truncated arcs, Topology, 41 (2002), 1031-1040.

[4] J. Gordon, Motivic Haar measure on reductive groups, preprint, March 2002, available at math. AG/0203106.

[5] G. Guibert, Fonction zêta motivique associée à une famille de séries de deux variables, C. R. Acad. Sci. Paris Sér. I Math., 333 (2001), 457-460.

[6] H. Hosokawa, Igusa local zeta functions and parabolic castling transformation of prehomogeneous vector spaces, J. Number Theory, 74 (1999), 148-171.

[7] J. Igusa, On functional equations of complex powers, Invent. Math., 85 (1986), 1-29.

[8] J. Igusa, On the arithmetic of a singular invariant, Amer. J. Math., 110 (1988), $197-233$.

[9] J. Igusa, $b$-functions and $p$-adic integrals, Algebraic analysis, Vol. I, Academic Press, Boston, MA, 1988, pp. 231-241.

[10] J. Igusa, Local zeta functions of certain prehomogeneous vector spaces, Amer. J. Math., 114 (1992), 251-296.

[11] T. Kimura, The b-functions and holonomy diagrams of irreducible regular prehomogeneous vector spaces, Nagoya Math. J., 85 (1982), 1-80.

[12] A. Libgober, Hodge decomposition of Alexander invariants, preprint, October 2001, available at math. AG/0108018.

[13] E. Looijenga, Motivic Measures, Astérisque 276, Séminaire Bourbaki, exposé 874 (2002), 267-297.

[14] C. Sabbah, Modules d'Alexander et $\mathcal{D}$-modules, Duke Math. J., 60 (1990), 729-814.

[15] M. Saito, Modules de Hodge polarisables, Publ. Res. Inst. Math. Sci., 24 (1988), 849-995.

[16] M. Saito, Mixed Hodge modules, Publ. Res. Inst. Math. Sci., 26 (1990), 221-333.

[17] M. Sato and T. Kimura, A classification of irreducible prehomogeneous vector spaces and their relative invariants, Nagoya Math. J., 65 (1977), 1-155.

[18] M. Sato and T. Shintani, On zeta functions associated with prehomogeneous vector spaces, Ann. of Math., 100 (1974), 131-170.

[19] Y. Teranishi, Relative invariants and b-functions of prehomogeneous vector spaces $\left(G \times \operatorname{GL}\left(d_{1}, \ldots, d_{r}\right), \tilde{p}_{1}, M(n, C)\right)$, Nagoya Math. J., 98 (1985), 139-156.

[20] J. Steenbrink, Mixed Hodge structures on the vanishing cohomology, Real and Complex Singularities, Sijthoff and Noordhoff, Alphen aan den Rijn (1977), 525-563.

[21] J. Steenbrink, The spectrum of hypersurface singularities, Théorie de Hodge, Luminy 1987, Astérisque, 179-180 (1989), 163-184.

[22] A. Varchenko, Asymptotic Hodge structure in the vanishing cohomology, Math. USSR Izvestija, 18 (1982), 469-512. 
[23] T. Yano and I. Ozeki, The b-function of a prehomogeneous vector space $\left(\mathrm{SL}(5) \times \mathrm{GL}(4), \Lambda_{2} \otimes \Lambda_{1}\right)$. Microlocal structure of the regular prehomogeneous vector space associated with $\mathrm{SL}(5) \times \mathrm{GL}(4)$. II, Research on prehomogeneous vector spaces (Kyoto, 1996), RIMS Kōkyūroku, 999 (1997), 92-115.

École Normale Supérieure

Département de mathématiques et applications

45 rue d'Ulm

75230 Paris Cedex 05

France

(UMR $8553 d u C N R S$ )

Francois.Loeser@ens.fr 